\title{
Radiofrequency current caused slowing of non- reentrant idiopathic right ventricular tachycardia originating from a wide arrhythmogenic area
}

\author{
Masaomi Chinushi, Yoshifusa Aizawa, Akira Shibata
}

\begin{abstract}
Radiofrequency catheter ablation was attempted in a patient with non-reentrant idiopathic right ventricular tachycardia (VT). Endocardial mapping indicated that the VT originated in the outflow tract of the right ventricle; however, an electrogram with an almost the identical activation time was recorded from an area extending to $1.0 \times 2.0 \mathrm{~cm}$. Each application of radiofrequency current within the area terminated VT, but a progressively slower VT with the same QRS configuration was induced until the area was covered by separate radiofrequency lesions. A progressive prolongation of VT cycle length might be related to a residual arrhythmogenic myocardium. Termination and slowing of the VT rate can be a hallmark of efficacy of each radiofrequency lesion.
\end{abstract}

(Br Heart f 1995;74:698-699)

Keywords: radiofrequency ablation; non-reentrant ventricular tachycardia; wide arrhythmogenic area

Idiopathic ventricular tachycardia of right ventricular origin has been successfully treated with radiofrequency catheter ablation. ${ }^{12}$

We reported on a patient in whom nonreentrant idiopathic ventricular tachycardia originated from a wide arrhythmogenic area in the outflow tract of the right ventricle. Radiofrequency currents applied within the area always terminated ventricular tachycardia, but until the whole area was covered by radiofrequency lesions a progressively slower ventricular tachycardia continued to be inducible.

First Department of Internal Medicine, Niigata University School of Medicine, Niigata, Japan M Chinushi

Y Aizawa

A Shibata

Correspondence to: Dr M Chinushi, First Department of Internal Medicine, Niigata Universit School of Medicine, 1-75 Asahimachi Niigata 951 , Japan.

Accepted for publication 25 April 1995.

A 48 year old woman was referred to our hospital with palpitation and fainting. Frequent non-sustained ventricular tachycardia was recorded on a 12 lead electrocardiogram. Treatment with conventional antiarrhythmic drugs did not prevent the recurrence of symptomatic non-sustained idiopathic ventricular tachycardia. During sinus rhythm the 12 lead electrocardiogram was normal. No underlying heart disease was found by standard examination.

After obtaining her informed consent we performed an electrophysiological study using the standard technique. Programmed electrical stimulation, which consisted of 1 to 3 extrastimuli and rapid pacing at cycle lengths between 667 and $286 \mathrm{~ms}$, applied at two sites in the right ventricle (apex and outflow tract) did not induce ventricular tachycardia. After isoprenaline was given intravenously nonsustained ventricular tachycardia with a configuration that was identical to that of the clinical idiopathic ventricular tachycardia occurred spontaneously. Ventricular tachycardia was also induced by rapid ventricular pacing at cycle lengths between 316 and $286 \mathrm{~ms}$ during isoprenaline administration (figure), but the cycle length of the ventricular tachycardia was constant at various pacing cycle lengths. Rapid pacing from the apex of the right ventricle did not produce transient entrainment. The outflow tract of the right ventricle was the earliest activation site for ventricular tachycardia. The local electrogram was normal in amplitude and width and occurred $30-40 \mathrm{~ms}$ before the onset of the QRS complex. However, an area with the same activation time extended $1.0 \times 2.0 \mathrm{~cm}$ around the origin. The best pace-mapping was obtained from this area.

While isoprenaline was administrated continuously, radiofrequency currents $(40 \mathrm{w})$ were applied to the site of ventricular tachycardia origin through a $7 \mathrm{Fr}$ quadripolar electrode catheter with a $4 \mathrm{~mm}$ large tip electrode. Ventricular tachycardia was terminated within seconds and did not recur during application of radiofrequency currents ( $30 \mathrm{~s}$ ). However, progressively slower ventricular tachycardia with the same $Q R S$ configuration continued to be induced until the area was covered by 16 separate radiofrequency lesions (figure). The systemic blood pressure and sinus rate were stable during radiofrequency ablation. No side effects were seen during or after ablation.

\section{Discussion}

In this patient ventricular tachycardia was induced by paced cycle lengths of between 316 and $286 \mathrm{~ms}$ during administration of isoprenaline but the rate of the ventricular tachycardia was not affected by the pacing 
A

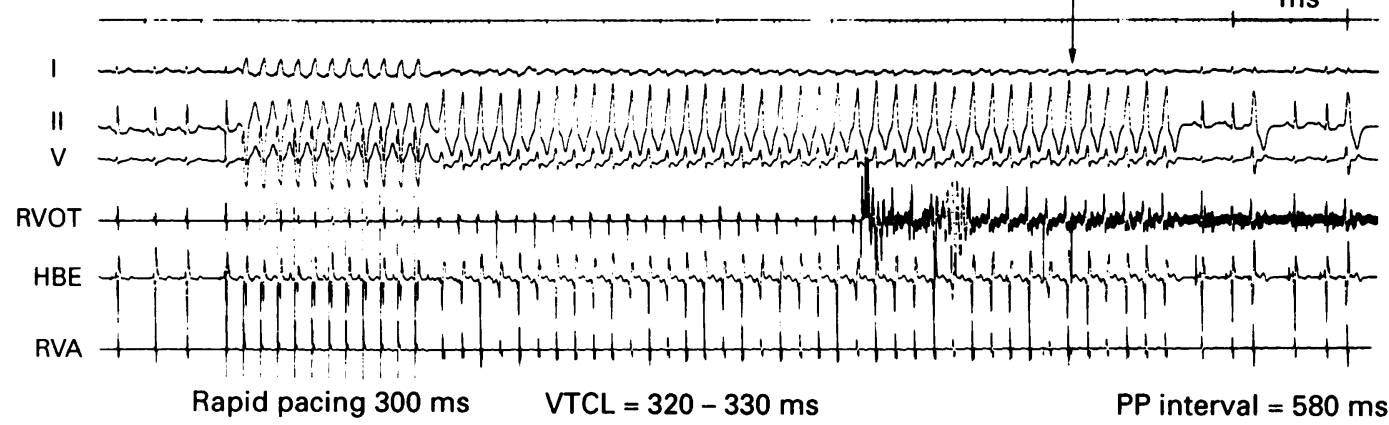

B

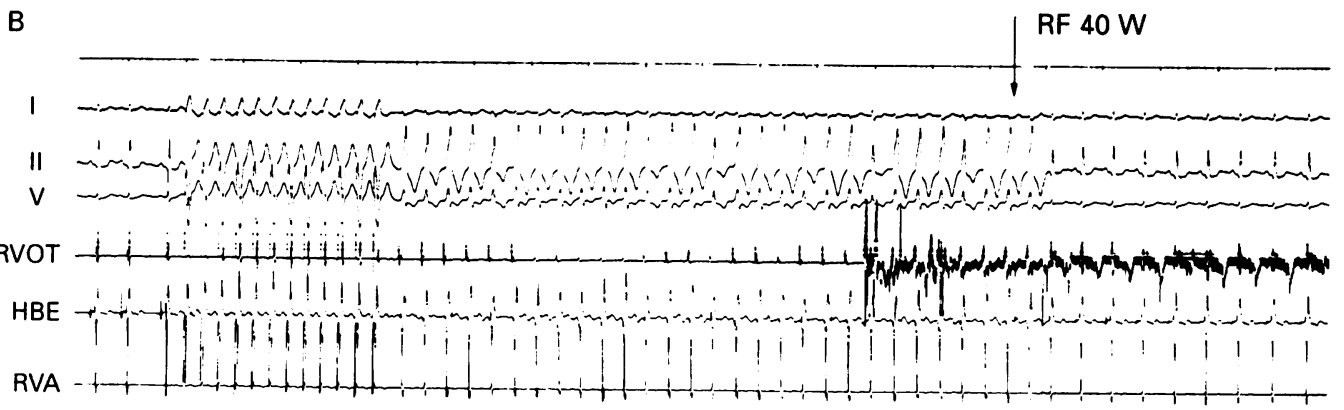

Rapid pacing $300 \mathrm{~ms} \quad \mathrm{VTCL}=380-400 \mathrm{~ms}$

PP interval $=580 \mathrm{~ms}$

Electrophysiologic findings. (A) Before radiofrequency ablation, rapid ventricular pacing at the cycle length of $300 \mathrm{~ms}$ induced ventricular tachycardia, and the cycle length of the ventricular tachycardia varied from 320 to $330 \mathrm{~ms}$. The earliest activation site of ventricular tachycardia was the outflow tract of the right ventricle, but isochronal presystolic eletrograms were obtained from an area extending $1.0 \times 2.0 \mathrm{~cm}$ around the site. Radiofrequency current (40 w) applied within the area terminated ventricular tachycardia, but slower ventricular tachycardia with the same $Q R S$ configuration remained inducible until the area was covered by 16 radiofrequency lesions $(B) . P P$ intervals did not differ during radiofrequency ablation. RVOT, outflow tract of the right ventricle; HBE, His bundle electrogram; RVA, apex of the right ventricle; VTCL, cycle length of ventricular tachycardia. See text for other abbreviations.

rate. Transient entrainment was not observed, and the local electrograms were normal at the site of ventricular tachycardia origin and in other segments of the right ventricle. These findings suggest a non-reentrant mechanism of the ventricular tachycardia, possibly abnormal automaticity.

An unusual finding in this patient was that as the number of radiofrequency applications increased, the ventricular tachycardia rate slowed progressively without changing the QRS configuration of the ventricular tachycardia. Sinus rate and systemic blood pressure were stable during radiofrequency ablation, so autonomic nervous tone was not an element in the slowing of the ventricular tachycardia rate.

In reentrant ventricular tachycardia, radiofrequency currents applied to the central common slow pathway might depress the conduction velocity and decrease the ventricu- lar tachycardia rate. ${ }^{2}$ Findings in our patient suggested an inverse relation between the residual mass of arrhythmogenic tissue and the ventricular tachycardia cycle length. The mechanism by which the radiofrequency lesion prolonged the ventricular tachycardia cycle length is not known. When ventricular tachycardia with the same configuration is terminated by each application of radiofrequency current but soon recurs, a progressive prolongation of the cycle length of the ventricular tachycardia might be used to predict the efficacy of radiofrequency ablation.

1 Klein LS, Shih HT, Hackett EK, Zipes DP, Miles WN Radiofrequency catheter ablation of ventricular tachyRadiofrequency catheter ablation of ventricular tachycardia in patients without
Circulation 1992;85:1666-74.

2 Aizawa Y, Chinushi M, Naitoh N, Kusano Y, Kitazawa H, Takahashi $\mathrm{K}$, et al. Catheter ablation with radiofrequency current of ventricular tachycardia originating from the right ventricle. Am Heart $\mathcal{F} 1994 ; 125: 1269-75$. 